\title{
New imaging techniques in the treatment guidelines for lung cancer
}

\author{
C. Schaefer-Prokop, M. Prokop
}

New imaging techniques in the treatment guidelines for lung cancer. C. SchaeferProkop, M. Prokop. (C) ERS Journals Ltd 2002.

ABSTRACT: Computed tomography (CT) remains the main imaging technique for the preoperative staging and post-therapeutic evaluation of bronchogenic carcinoma. Spiral CT has already overcome some of the problems encountered with central or more extensive tumours. Multislice CT offers further improvement and allows for scanning of the whole chest within a single breath-hold using a thin-section high-resolution technique. Problem-adapted sections in arbitrary directions become available and provide an excellent spatial resolution. One can expect improved accuracy for the evaluation of transfissural tumour growth, chest wall involvement, mediastinal infiltration and lymph node staging.

Despite recent advances in magnetic resonance (MR) techniques for imaging the chest, the role of MR for staging of bronchogenic carcinoma remains limited. It offers advantages such as the assessment of chest-wall involvement or mediastinal involvement in patients in whom CT remains equivocal. Lymph-node-specific MR contrast agents offer new diagnostic potential for the assessment of metastatic disease.

New techniques for the display of three-dimensional data sets include volume rendering and virtual bronchoscopy. These techniques represent new tools for the evaluation and demonstration of pathology within the central tracheobronchial tree. Their most important application is the guidance of bronchoscopic biopsies.

The assessment of an indeterminate pulmonary nodule is frequently based on positron emission tomography imaging. As an alternative, nodule vascularization (contrast enhancement patterns on CT or magnetic resonance imaging (MRI)), calcifications (absorption characteristics at various X-ray energies on CT or dual energy radiography), and morphological features (high resolution imaging at $\mathrm{CT}$ ) can be used as the basis for nodule differentiation. The dynamics of contrast enhancement in CT or MRI can also be used for the assessment of tumour viability after chemotherapy.

Lung cancer screening programmes are still controversial. Low-dose computed tomography scanning and computed assisted detection algorithms based on chest radiographs or computed tomography scans form the technical basis for such projects. Eur Respir J 2002; 19: Suppl. 35, 71s-83s.
Dept for Radiology, University of Vienna, Austria.

Correspondence: C. Schaefer-Prokop

Dept of Radiology

University of Vienna

Vienna General Hospital

Währinger Gürtel 18-20

A 1090 Vienna

Austria

Fax: 431404004894

E-mail: Mathias.Prokop@univie.ac.at

Keywords: Low-dose computed

tomography

lung cancer

positron emission tomography oncology

spiral computed tomography

virtual bronchoscopy

Received: September 92001

Accepted September 282001
Lung cancers are estimated to account for $\sim 15 \%$ of all cancers and represent the leading cause of death from cancer. The mainstay of lung cancer therapy of nonsmall cell lung cancer is still surgery and the strongest prognostic indictor for survival is whether or not the cancer can be completely resected.

Computed tomography (CT) is still the imaging modality of choice in studying the tumour itself and detecting lymph node and extrathoracic metastases. The aim of preoperative assessment of patients is to avoid unnecessary thoracotomy in patients with unresectable disease while allowing patients with potentially resectable lung cancer to benefit from surgery. While staging of small peripheral lung cancers (T1) or obviously advanced central tumours (T4) is relatively straightforward, the differentiation between locally advanced but potentially resectable tumours (stage IIIa) or locally advanced but unresectable tumours (stage IIIb) represents a real challenge for any imaging modality. The limitations of CT staging in lung cancer are well recognized and mainly result from the uncertainty of detecting metastases in normal-sized lymph nodes and from the difficulty in differentiating tumour adhesion from tumour infiltration.

In addition to the preoperative evaluation of patients, imaging techniques play a key role in monitoring and evaluating the effects of chemotherapy and radiation treatment. The differentiation of the remaining vital tumour from post-therapeutic scarring is essential in patients that may become eligible for a surgical approach.

With further improvement of technology, especially the development of low dose CT techniques, there has been a resurgent interest in screening for lung cancer. Preliminary data for limited studies are promising so far, but large multicentre trials are still ongoing to prove the advantages of mass lung cancer programme.

Magnetic resonance imaging (MRI) of the chest remains problematic even with the most recent scanners because of the vulnerability of MRI towards motion artifacts and a strong susceptibility to artifacts within the lung parenchyma. In addition, the lung 
parenchyma has a low intrinsic signal due to its low proton density.

Most recent CT and magnetic resonance (MR) techniques are characterized by an increased speed of scanning. This decreases the effects of motion and allows for a more efficient use of contrast enhancement. Multidetector spiral CT allows for volumetric scanning of the whole chest with excellent anatomical resolution. Thus images in arbitrary sectional planes can be obtained, a feature that used to be unique for MRI. Based on the volumetric data acquisition and elaborate data processing, virtual bronchoscopic views of the central tracheobronchial system can be produced.

Chest radiography remains the preferred initial imaging technique for patients with known or suspected lung cancer because of its availability, low cost, low radiation dose and diagnostic sensitivity. Advanced processing of digital radiographical data may be used as front-end for automatic lesion detection that was found to be helpful in the detection of subtle lesions that are easily overlooked due to overlying anatomical structures.

This review article presents the most recent technical developments of radiographical and tomographical imaging techniques including CT and MRI, with special emphasis on their impact on staging of patients with bronchial carcinoma. The accuracy of modern CT techniques using thin-section scanning or multidetector technology in the assessment of specific aspects of the tumour, node, metastasis (TNM) classification, such as mediastinal or chest-wall involvement, the differentiation of benign versus malignant intrapulmonary nodules, or the assessment of malignant lymphadenopathy, will be reviewed. New options of modern MR technology are presented. Options and limitations of these techniques will be discussed, supported by a review of the most recent literature and illustrated by a selection of images.

\section{Technical advances in digital radiography}

Digital radiography detectors had already been introduced in the early eighties in the form of storage phosphor plates. They represent a cassette-based system that could be applied in all existing radiographical equipment. Other detector types followed in the form of dedicated chest units which used selenium as a detector material, or in the last $1-2$ yrs as a flat panel or direct detector technique. All digital detectors in common have a wider dynamic range as compared to the conventional film that allows for a superior capture of the wide attenuation differences within the lung and an automatic optimization of contrast and image density providing a more constant image quality irrespective of exposure variations (within a certain range) in common.

\section{Temporal subtraction}

Most important with respect to the detection of focal lung lesions are new processing options such as temporal subtraction and dual energy subtraction that represent the front-end towards algorithms for computer-aided diagnosis (CAD) [1]. Temporal subtraction is based on a subtraction of two posteroanterior chest radiographs taken at two different points in time. Ideally, the subtraction process can reveal subtle interval changes in the radiographs because it removes all features that are constant over time. Precondition is an elaborate algorithm for automated registration of the two images that reduce subtraction artifacts due to varying inspiration depth or changing radiological projection. So far, this makes this processing option only available in dedicated imaging centres and a limited number of patients in whom the follow-up radiographs are similar enough to be registered.

The subtracted images have been shown to reveal subtle parenchymal changes that may have been overlooked due to overlying and distracting anatomical structures (e.g. bones, vascular structures).

\section{Dual-energy subtraction}

Dual-energy subtraction uses the different absorption characteristics of calcified and noncalcified structures as a function of exposure voltage [2]. The technique uses either a fast double exposure at varying $\mathrm{kVp}$ (usually 80 and $120 \mathrm{kVp}$ ) or a single exposure technique with a metal filter between two detector screens. As a result of both techniques, images are obtained with high and low X-ray energy. Low-energy images display calcifications more prominently while high-energy images relatively suppress calcifications. Weighted subtraction of the two digital data sets yields images that predominantly show either softtissue structures (lung parenchyma without overlying ribs) or bone structures (calcified nodules, vertebrae and ribs). Initial limitations of image quality due to increased image noise could be overcome by special postprocessing techniques for noise reduction. Chest units with dual-energy subtraction are in clinical practice now with both detector types, storage phosphor plates (Fuji, Tokyo, Japan) as well as with flat panel technology (GE, Milwaukee, MN, USA).

Dual energy subtraction has been shown to improve detection of pulmonary nodules [2] and may improve lung cancer screening using conventional X-rays.

\section{Computer-aided diagnosis}

CAD performs an automated analysis of the image, using criteria developed from large numbers of normal and abnormal cases [3]. Complex computer software is necessary to enable the software to distinguish between an abnormal focal abnormality and normal anatomical background. The idea is to direct the radiologist's attention towards a suspicious area in the image. The average improvement in accuracy has been substantial and depends on the readers' experience as well as on the complexity of the task (e.g., interstitial disease versus nodule detection). 


\section{Technical advances in computed tomography}

\section{From single detector computed tomography to multidetector computed tomography}

The technique of spiral CT was introduced in 1989 and replaced the discontinuous acquisition of data in conventional CT by a volumetric data acquisition. It is based on continuous rotation of the tube around the patient, who is transported through the scanning plane with a constant table feed $\cdot \mathrm{s}^{-1}$. The table feed may be increased by a factor of two (the so-called pitch factor) over the slice collimation, making it possible to cover larger scan ranges in one breath-hold than with a conventional scanner and at the same time reducing radiation exposure.

While the spatial resolution in the scanning plane is high (pixel size of $0.6-0.8 \mathrm{~mm}$ in chest CT), the resolution along the patient axis (z-axis) is markedly lower (5-10 $\mathrm{mm}$ in standard scanning protocols). The $\mathrm{z}$-axis resolution is increased, with narrower slice collimation. The volume that has to be covered within one breath-hold, of some $30 \mathrm{~s}$, limits how narrow the slice collimation can be. With thin collimations of 1-2 mm a range of only $6-12 \mathrm{~cm}$ can be covered. As a consequence, with single detector spiral CT, thinsection scanning had to be focused on the region of obvious pathology.

The z-axis resolution can be improved by using faster scanners with an increased speed of tube rotation. In the early $1990 \mathrm{~s}$ only $1 \mathrm{~s}$ scanners were available. They were followed by $0.75 \mathrm{~s}$ scanners in the mid 1990s, and $0.5 \mathrm{~s}$ scanners at present. Faster scanners increase volume coverage with thinner sections by up to a factor of 2 ( $0.5 \mathrm{~s}$ scanners).

Further improvement had been introduced in the early nineties with the advent of split detector systems. In these CT scanners, two parallel detector rows were exposed simultaneously. Such scanners were able to acquire two images at once thus doubling scanning speed. Although providing superior technology they were only slowly accepted.

In 1998, four of the major vendors announced a breakthrough in CT technology that was based on a complex array of parallel detector rows that could be simultaneously exposed. This technology was termed multislice CT, multidetector computed tomography (MDCT), multidetector-row CT, and quite a few more terms. With present scanners four CT slices can be acquired simultaneously, and most scanners apply subsecond tube rotation of $0.5-0.8 \mathrm{~s}$. As a result the performance of these scanners as compared to singleslice spiral CT is increased by a factor of $\leqslant 8$. This makes it possible to either scan the chest with $1-1.25 \mathrm{~mm}$ thick sections in $20-30 \mathrm{~s}$, or to scan it with $2-2.5 \mathrm{~mm}$ sections in $10-60 \mathrm{~s}$.

\section{Multidetector computed tomography scanning procedure}

The tracheobronchial system should be free of any mucus because CT often cannot differentiate between a focal collection of mucus and a tumour or polypoid lesion. Thus, the patient should be encouraged to cough immediately prior to the exam.

Scanning in a caudocranial direction is useful because it reduces the amount of breathing artifacts if the patient can no longer hold the breath close to the end of the scan.

Advanced contrast material administration. Depending on the scan duration, a total of some $90-120 \mathrm{~mL}$ of contrast material should be injected with $3-4 \mathrm{~mL} \cdot \mathrm{s}^{-1}$ in order to get a high contrast enhancement. For the staging of lung cancer a start delay of some $30 \mathrm{~s}$ can be recommended because there will be better differentiation between collapsed lung and tumours. Individualized bolus injections may become feasible soon (either derived from a test bolus or mathematical modelling).

After contrast material administration, a saline flush should be employed that pushes the contrast medium forward and flushes the injection veins. As a result, the contrast material is better utilized. Also the amount of artifact in the brachiocephalic veins is reduced when caudocranial scanning is employed.

Optimized scanning parameters. Multidetector CT scanners can now cover a range of $>24 \mathrm{~cm}$ in $<30 \mathrm{~s}$ even though $1 \mathrm{~mm}$ or $1.25 \mathrm{~mm}$ sections are employed. Such protocols are to be preferred for the staging of bronchogenic cancer because they provide superior detail assessment and the opportunity for imaging in any arbitrary plane. In addition, such near-isotropic data sets allow for optimum three-dimensional (3D) renderings.

The effective thickness of a section ranges between $1.25-1.6 \mathrm{~mm}$ with these protocols. Overlapping reconstruction every $0.6-1 \mathrm{~mm}$ will yield a near-isotropic set of 3D data points that serves as a "secondary raw data set" from which other sections in arbitrary planes can be reformatted. The thin sections substantially reduce partial volume averaging and thus improve the display of fine structures [4].

Image evaluation. A near-isotropic data set from multislice CT consists of 250-450 images that are hard to review interactively on most workstations. In addition, these images may suffer from increased image noise, especially in obese patients. If one does not want to increase radiation exposure to the patient, increasing the thickness of the chosen imaging plane can also reduce image noise.

For the staging of peripheral bronchogenic cancer, $1.5-\mathrm{mm}$ thick sections in a sagittal and coronal plane should be reformatted in the area of the suspected tumour in order to optimally assess its relation to the pleura, interlobar fissures, vessels and bronchi. For the mediastinum, for central tumours or tumours abutting the chest wall or superior sulcus, 2-4 mm wide sections can be reformatted in a plane that is perpendicular to the interface to the tumour. For mediastinal lymph nodes, $3-5 \mathrm{~mm}$ wide axial and coronal sections can be recommended. The use of a 
CT workstation for image interpretation becomes highly advisable [5].

Multislice CT produces excellent images that exceed most MRI sections in quality (figs. 1 and 2). Similar to MRI, coronal and sagittal image sets may even serve as a primary mode of image interpretation.
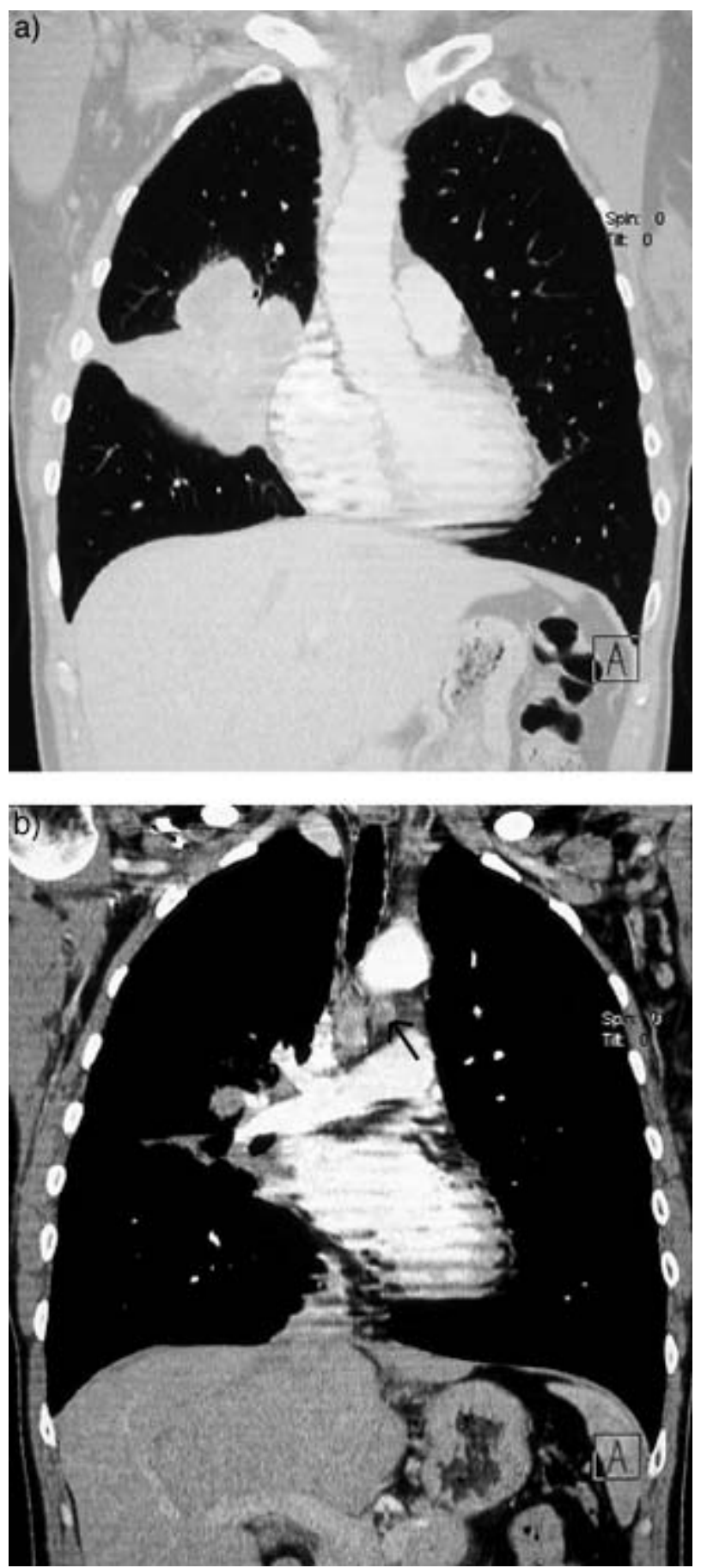

Fig. 1.-a) Coronal reformations of a multidetector computed tomography data set in a patient with broad transfissural tumour growth. b) Pathological ipsilateral hilar and mediastinal lymph nodes and a small but round pathological lymph node in the aortopulmonary window (arrow) that defines N3 disease and thus irresectability.

\section{Virtual bronchoscopy and computed tomography bronchography}

Virtual bronchoscopy is a $3 \mathrm{D}$ visualization technique in which a CT data set is used to create images of the inner surface of the tracheobronchial system [6] (fig. 3). Virtual bronchoscopy requires perspective rendering techniques and various interaction tools to obtain the visual impression that the observer is actually moving within the data volume.

The technique can be used to noninvasively assess anomalies of the central tracheobronchial system but cannot substitute for fibreoptic bronchoscopy in patients in whom biopsy has to be performed.

In addition to virtual bronchoscopy, other rendering techniques can be used to display the tracheobronchial system (CT bronchography). They employ either a shaded display of the luminal surface (surface shaded display (SSD)) or present the tracheobronchial anatomy as 3D structure with semitransparent walls (volume rendering techniques (VRT)).

As a prerequisite of virtual bronchoscopy or CT bronchography, the CT data set should be as highly resolved as possible, and should be subject to no misregistration artifacts due to motion between adjacent transaxial sections. This became possible with spiral CT scanning but multislice CT scanners are much superior; scanning is faster, and the spatial resolution in the longitudinal direction can be markedly improved. While spiral CT was able to gain excellent results for the trachea and central bronchi, multislice CT expands the application to further distal bronchial segments. For optimum image

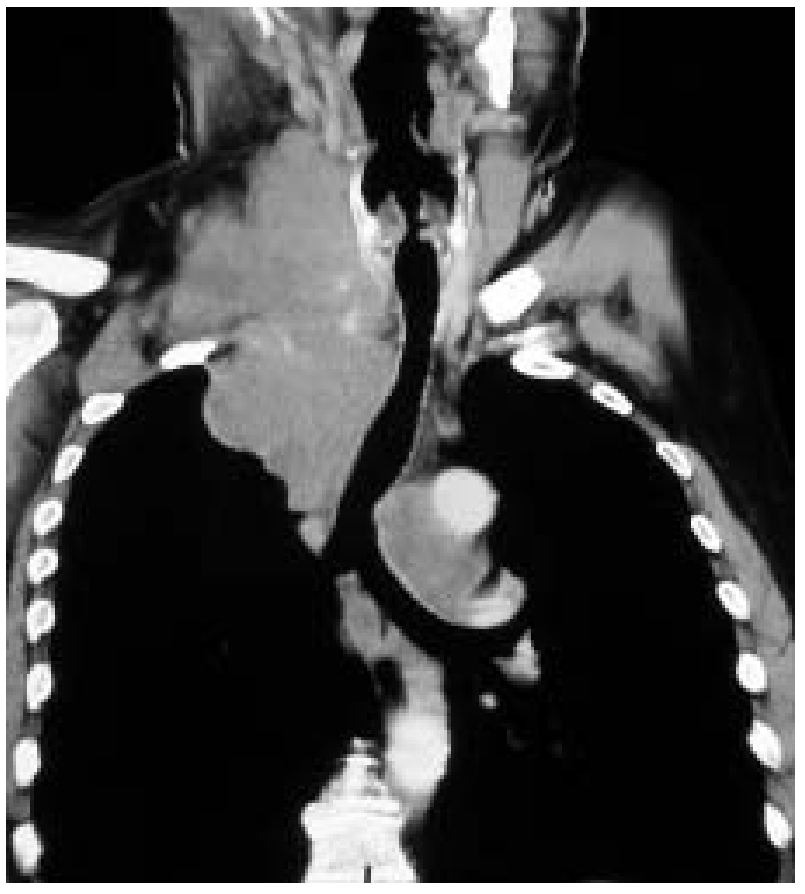

Fig. 2.-Coronal multidetector computed tomography view of a patient with a Pancoast tumour that has broadly infiltrated the neck including the cervical vessels and the mediastinum including the aorta. There are pathological lymph nodes in the aortopulmonary window and the subcarinal area. 

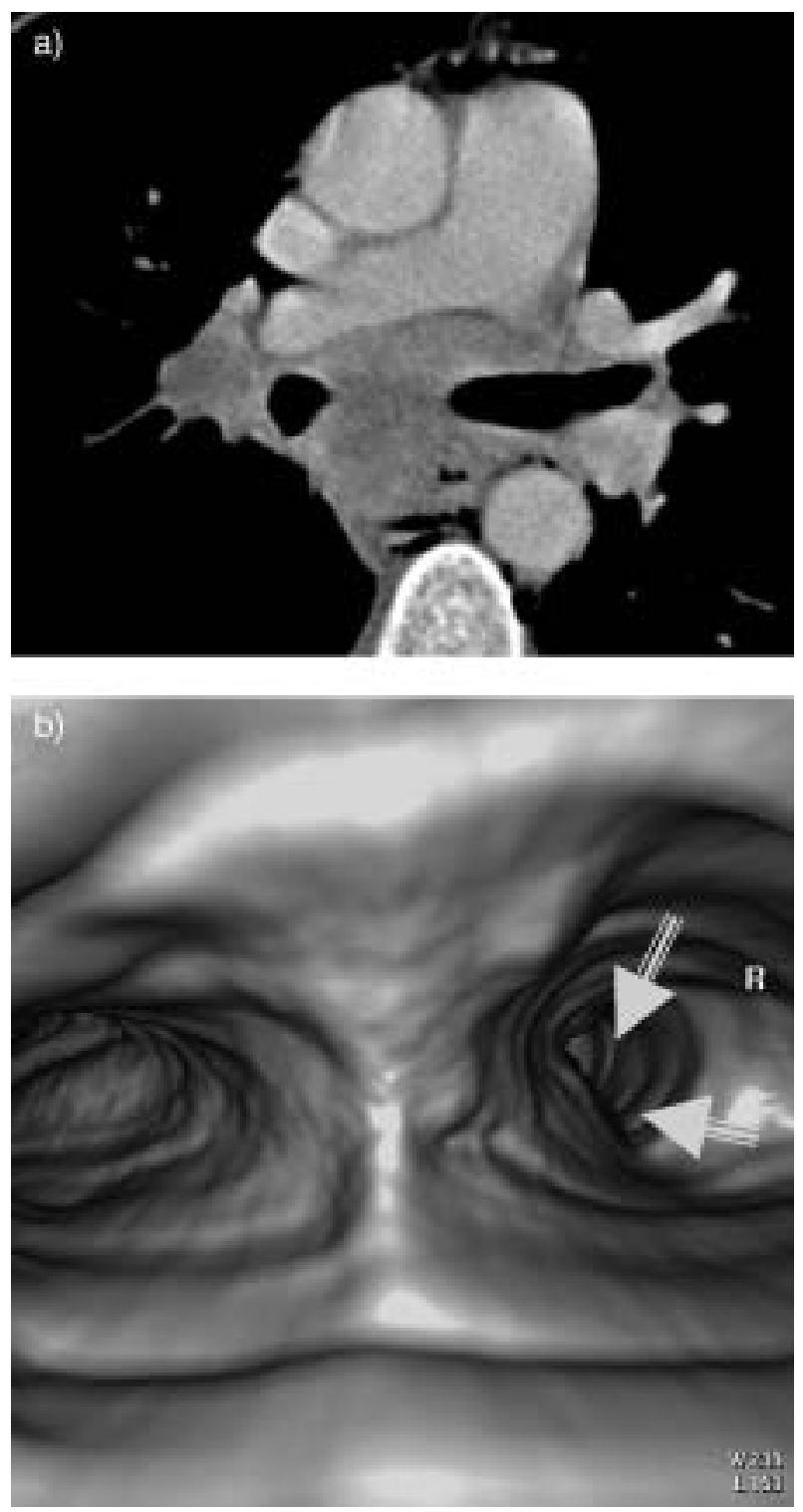

Fig. 3.-a) Computed tomography section and b) virtual bronchoscopic view in a patient with a mediastinal tumour infiltrating the medial wall of the right main bronchus (arrow). Images courtesy of P. Rogalla, Berlin, Germany.

quality, motion artifacts due to pulsation or breathing have to be minimized [7].

Due to the perspective distraction, measurements should not be performed on the endoscopic views but on the initial two-dimensional (2D) data or by using multiplanar reformations [8].

\section{Technical advances in magnetic resonance imaging}

Despite continuous improvements in examination technique and image quality, MRI has not yet become established as a routine procedure in the diagnosis of thoracic tumours. This is due to the high susceptibility of MRI to motion artifacts (breathing and pulsation), the intrinsic low proton density of lung parenchyma and the further decrease of signal by strong susceptibility artifacts due to the multiple airsoft tissue interfaces within the lung.

CT, especially the new CT techniques, represents the imaging technique of choice for the delineation of the lung parenchyma. Nevertheless, MRI may be indicated as a "problem solving tool" in studies of the mediastinum and the chest wall. The specific advantages of MRI include primary multiplanar imaging, excellent soft tissue contrast and delineation of tumours and vessels without the use of contrast media.

\section{Breath-hold imaging and improved breathing corrections}

Usually T2-weighted turbo spin-echo (TSE) and T1-weighted spin echo (SE) sequences are performed before and after administration of gadolinium chelates.

To cope with respiratory motion, some of the recently developed scanning sequences (fast low-angle shot (FLASH) or half fourier turbo-spin echo (HASTE)) can be acquired in one breath hold with acquisition times well below $30 \mathrm{~s}$. For nonbreath-hold MRI, however, gating procedures are indispensable.

Navigator techniques with registration of the diaphragm [9], gating in expiation using a belt or respiratory compensation using reordering of phaseencoding lines of the $\mathrm{k}$-space (reordering of phase encoding (ROPE) or phase encoding and reordering (PEAR)) were described. Respiratory gating should be complemented by diastolic electrocardiogram (ECG) triggering $[10,11]$. Prospective respiratory gating is recommended for T2-weighted fast spin echo (TSE) sequences, while respiratory compensation techniques such as ROPE have proven effective in T1-weighted sequences [12].

For evaluation of chest-wall involvement MRI offers excellent soft-tissue contrast. Image quality further benefits from using dedicated surface coils instead of the body coil. Breath-hold sequences with a data acquisition time of few seconds in inspiration and expiration allow for the assessment of mobility of pleural adherent tumours for differentiation of adherence versus infiltration (see later).

\section{Reducing susceptibility effects of the lungs}

To make use of the higher proton density in opacified lung parenchyma, and to reduce susceptibility artifacts in normal, aerated lung parenchyma, different approaches have been developed that are based on the use of short echo times or ultrashort echo times with projection-reconstruction techniques $[13,14]$ to reduce signal loss from T2 relaxation resulting in a significant improvement of the signal to noise ratio [15] (fig. 4). These approaches include: 1) T1-weighted $\mathrm{SE}$ sequences with short echo times of $\mathrm{TE}<7 \mathrm{~ms}$ [15]; 2) T1-weighted gradient echo sequences (GRE) with short TE times (FLASH) of $3 \mathrm{~ms}$ or a higher number of acquisitions; and 


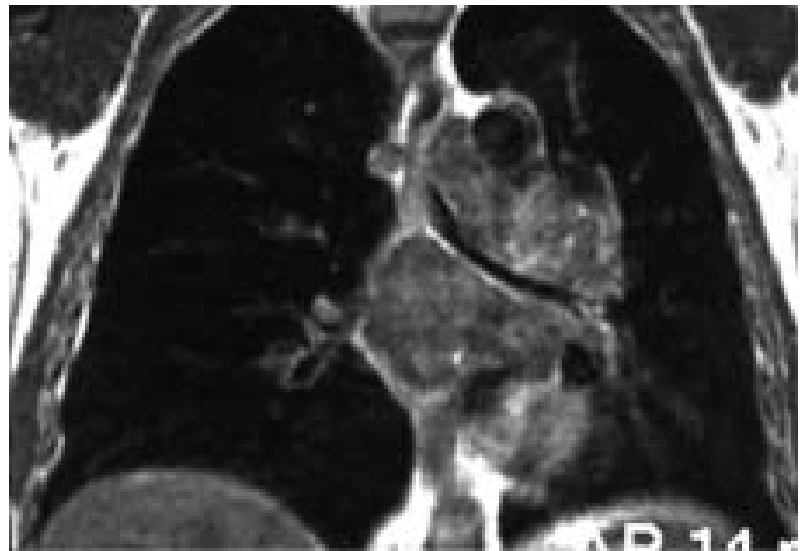

Fig. 4.-Coronal Gd-enhanced T1-weighted magnetic resonance section in a patient with a T4 bronchial carcinoma that has infiltrated the aortic arch, the pulmonary artery, the left main bronchus and the trachea (image courtesy of P. Landwehr, Dept of Radiology, Henriettenstiftung, Hannover, Germany).

3) T2-weighted TSE or T2-weighted ultrafast TSE sequences with high turbo factors (UTSE).

In addition, iatrogenic increase of the relaxivity of existent spins or enrichment of spin density is achieved by administration of contrast agents such as gadolinium chelates for enhancing nodules or opacifications. Oxygen enhancement or the administration of hyperpolarized noble gases can be used for ventilation imaging.

\section{New magnetic resonance contrast agents}

New MR contrast agents with ultrasmall iron oxide particles are currently under investigation. In normal lymph nodes with functioning macrophages the iron oxide particles are phagocytosed and thereby decrease the signal intensity on MRI. Metastatic nodes, lacking macrophages, do not take up the contrast agent and hence show no change in signal on postcontrast images [16]. These agents were shown to increase sensitivity for nodal metastasis by detection of metastasis in normal-sized nodes in pelvic tumours [17]. Clinical trials in patients with bronchial carcinoma are currently underway.

\section{Detection of bronchogenic carcinoma}

\section{Computer-aided diagnosis and image enhancement techniques}

CAD includes all approaches that apply computer techniques to radiological diagnostic decision making. CAD may either support lesion detection or may help the radiologist to associate different underlying diseases to a certain detected lesion pattern [3].

CAD may be improved if the input images used for CAD algorithms already have the components of one image separated (dual energy subtraction) or have information of different images integrated (temporal subtraction). Both techniques are especially interesting in the context of using chest radiography as a screening procedure to detect subtle focal intrapulmonary lesions, which are frequently obscured by overlying anatomical structures such as vessels or ribs. Only energy subtraction is currently commercially available; all other approaches are still confined to a research environment.

Since dual-energy subtraction separates the calcium and soft tissue components of the thorax, it was shown to significantly improve the detection of focal pulmonary lesions, like early bronchogenic cancer, especially when obscured by overlying bones. While the soft tissue images improve detection of pulmonary nodules, bone images are useful for confirming the presence of calcifications in benign nodules or mediastinal lymph nodes, and for improving visualization of bone destruction or calcified pleural plaques [2].

Temporal subtraction of two follow-up radiographs is suited to highlight areas of subtle change that may not be obvious on the single radiograph. In experimental studies it was shown to significantly improve the detection of a broad range of abnormalities including nodules, infiltrative disease or alterations of the hilar or mediastinal shadow [1]. The technique is still under investigation for its appropriateness in a lung cancer screening program in Japan.

CAD programs have been introduced for radiography as well as for CT scans and have been shown to improve detection rates considerably [3]. They are likely to play an increasing role in patients at risk for developing lung cancer or metastases.

\section{Screening with low-dose computed tomography}

Previous studies using chest radiography and sputum cytology had not been able to statistically prove reduction of mortality rates of bronchogenic cancer. This was most likely because these tests were not sensitive enough to detect early tumour stages.

Spiral CT is acknowledged as the most sensitive diagnostic method for the detection of small intrapulmonary nodules [18]. The option for significant reduction of acquisition dose without compromising the conspicuousness of intrapulmonary nodules makes this technique technically feasible for screening. Appropriate choice of parameters allow for CT examinations having a sensitivity exceeding $90 \%$ for nodules $>5 \mathrm{~mm}$ in diameter with an acquisition dose slightly higher than a chest radiograph in two projections [19].

Due to the large number of nodules with benign histology (granuloma, hamartoma, focal infection, lymph nodes) additional diagnostic tools have to be defined to differentiate benign from malignant focal lesions. Computed algorithms based on 3D display and volumetric assessment can detect even subtle growth of a pulmonary lesion. Such algorithms were found to reliably assess volumetric changes after as little as 4 weeks [20].

At present there a numerous studies under way or in the planning phase. The most important ones to date are the Early Lung Cancer Action Project (ELCAP) trial in New York City, and two Japanese multicentre trials [21-23]. In all of the studies published so far 
CT was found to be vastly superior to conventional radiography in terms of sensitivity. The initial CT screening detected bronchogenic carcinoma in 1.1$2.7 \%$ of screened patients. Between $62-93 \%$ of tumours were determined to be stage I [21-23].

The relatively high rates of slowly growing adenocarcinomas in the ELCAP trial as well as in the two Japanese trials suggest a bias for the detection of slowly growing cancer types (length time bias). Incidence screening by follow-up CT could detect a lower rate of additional cancers [24] and there were more aggressive tumour types diagnosed in the time intervals between two screening procedures. This holds true especially for the more frequently centrally located small cell carcinomas. In the Japanese multicentre trials primary detection failures (lesions that were found by follow-up but could be retrospectively seen on the initial screening exam) could be shown to be due to the misinterpretation of lesions in close vicinity to pulmonary vessels or associated with preexisting disease due to old tuberculosis [25]. Other criticism refers to the fact that a nonrandomized trial without inclusion of a control group does not allow for the quantification of factors such as lead time bias, length bias and overdiagnosis bias [24].

\section{Differentiation of pulmonary nodules}

Evaluation of the solitary pulmonary nodule remains a substantial challenge in modern medicine. Approximately $50 \%$ of surgically-removed nodules turn out to be benign [26].

\section{Dual-kilovolt peak computed tomography}

Preliminary in vitro and in vivo work indicated that dual-kilovolt peak CT might be useful in the identification of benign lesions. CT numbers of benign lesions should be higher on $80 \mathrm{kVp}$ images than on $140 \mathrm{kVp}$ images based on differences in calcium content between benign and malignant nodules. However, this could not be confirmed in a multicentre trial that included 157 lesions with histological proof [27]. Measurements were performed on 3-mm thick slices that were obtained in spiral CT technique with highly overlapping image reconstruction over a scan length of $15 \mathrm{~mm}$.

\section{Contrast enhancement characteristics}

Contrast enhancement characteristics after intravenous bolus injection of contrast material can help differentiate benign from malignant disease.

A recent study by SwENSON et al. [28] found that absence of significant lung-nodule enhancement $(\leqslant 15$ Hounsfield units (HU)) at CT was strongly predictive of benign disease. In this study, a $15 \mathrm{~mm}$ long spiral CT scan of 3-mm thick slices was obtained 1, 2, 3 and 4 min after the onset of injection of contrast material (420 mg iodine $\cdot \mathrm{kg}^{-1}$ body weight) with an injection rate of $2 \mathrm{~mL} \cdot \mathrm{s}^{-1}$. At a concentration of $300 \mathrm{mg}$ iodine $\cdot \mathrm{mL}^{-1}$ between $50-175 \mathrm{~mL}$ of contrast medium were injected depending on the body weight. Data analysis was based on peak nodule enhancement and time attenuation curves. Malignant lesions enhanced significantly more (median of $38 \mathrm{HU}$ with a range 14-165 HU) than benign lesions (median of $10 \mathrm{HU}$ with a range of $-20-96 \mathrm{HU}$ ). The multicentre trial included 356 nodules, of which $48 \%$ were malignant. The size of the nodules ranged $14-55 \mathrm{~mm}$ with a median of 13 and $17 \mathrm{~mm}$, respectively, for the benign and malignant lesions. With a threshold of $15 \mathrm{HU}$ the sensitivity was $98 \%$ (167 of 171 malignant nodules), the specificity was $58 \%$ (107 of 185 benign nodules) and the accuracy was $77 \%$ (274 of 356 nodules) [28].

The lung nodule enhancement protocol was successfully carried out in combination with an upper abdominal CT scan (obtained between the first and second spiral scan after onset of injection) or a complete thoracic CT examination in patients that had been referred for complete staging.

ZHANG et al. [29] reported corroborating results. They found that peak enhancement levels were significantly higher for malignant (42 HU) and inflammatory benign lesions (44 HU) than for noninflammatory benign nodules (13 HU). Nodules with active inflammatory reactions were controlled after treatment (3-4 months later) and showed significantly reduced enhancement rates that made discrimination possible. They pointed out that intratumoral necrosis and low cardiac output may contribute to low peak enhancement and thus false negative evaluation. ZHANG et al. [29] used a higher injection rate of $4 \mathrm{~mL} \cdot \mathrm{s}^{-1}$ and acquisition of a cluster of ten scans of $5 \mathrm{~mm}$ thickness in a dynamic fashion after 15, 75 and $135 \mathrm{~s}$.

As a further tool for discrimination between malignant and inflammatory nodules the authors described a delayed washout of the contrast medium from malignant nodules. They found a slower decline of the density curves in malignant nodules, and a lower attenuation on precontrast scans in inflammatory nodules.

The high predictive value for benign disease is potentially valuable in the treatment of patients. A nonenhancing lung nodule can be managed with radiological surveillance alone, even in a supportive clinical situation. An enhancing nodule will be either appropriately followed if clinical suspicion for malignancy is low or will undergo biopsy.

Similar results have been reported for MRI as well. Gd-enhanced dynamic scanning is used with MRI at similar time intervals as in CT [30].

\section{Volumetric analysis of nodule growth}

Observation of nodule growth is another valuable tool for monitoring pulmonary nodules, especially if they are too small to be biopsied or assessed by enhancement characteristics, and if they occur in patients at risk. Computer programs were developed that allow for nodule segmentation with automated volume measurements as well as for nodule visualization using modern volume rendering techniques. Such 
programs proved to be much more precise and sensitive towards small volume changes than visual assessment of nodule growth or manual measurements of lesion diameter on transaxial CT sections [20].

\section{Positron emission tomography}

It is important to note that positron emission tomography (PET) achieves a sensitivity for the detection of malignancy in nodules that is comparable to the reported values for enhancement characteristics (94-100\% versus 98\%). However, specificity with PET is superior $(>90 \%)$ to the numbers with contrast dynamics $(60-75 \%)$. The high specificity of ${ }^{18} \mathrm{~F}$-fluoro2-deoxy-glucose (FDG)-PET is clinically important for the diagnosis of benign lesions. Lesions with low FDG uptake may be considered benign. There are false-positive results, however, for carcinoid tumours, bronchoalveolar carcinomas, and if lesions are $<1 \mathrm{~cm}$ [31].

As a consequence, small lesions are best followed by volumetric analysis of nodule growth, while larger nodules may be evaluated either by PET or dynamic contrast enhancement. Negative results in PET should be followed radiographically in order not to miss carcinoid tumours or bronchoalveolar carcinomas.

\section{Radiological staging of bronchogenic cancer}

Improved results for clinical staging of bronchogenic carcinoma with $\mathrm{CT}$ were described for thinsection scanning using dynamic CT, spiral CT or electron beam CT. Multidetector CT can be expected to substantially improve the evaluation of more advanced tumour stages. The relation of a tumour to the pulmonary fissures or transfissural growth is exquisitely well demonstrated (fig. 1). The evaluation of invasion of the chest wall or mediastinum can be expected to benefit as well. Due to its excellent spatial resolution along arbitrary imaging planes, MDCT can be expected to outperform MRI for imaging tasks including the evaluation of superior sulcus tumours.

Presently MRI is not superior but, depending on scanner technology and patient cooperation, is often inferior to $\mathrm{CT}$ for almost all indications. It may serve as a problem-solving tool in selected cases with regard to early chest wall involvement or the determination of mediastinal involvement. Interesting new possibilities are arising with regard to the use of lymph-node specific contrast agents, whose performance is not yet determined.

PET scanning will probably remain superior when it comes to evaluation of tumour spread to the lymph nodes, pleura and distant sites. MDCT, however, will substantially aid in choosing the right targets for further interventions, and will remain the most important tool for surgical planning.

\section{T-staging}

Staging of T1 and T2 tumours is based almost exclusively on CT scanning. Results are excellent, especially when thin-section imaging is employed. The situation becomes more difficult with T3 and T4 tumours even though the differentiation is critically important for determining whether or not a patient is a candidate for surgery.

Mediastinal invasion. Both CT and MRI have the same limitations in distinguishing tumour contiguity from tumour invasion into mediastinal structures. The prespiral CT era reported sensitivity values of only $55 \%$, or even lower for the correct staging of mediastinal invasion and the differentiation of stage IIIa versus IIIb [32, 33]. The superiority of MRI was due to the superior delineation of mediastinal fat (bright in T1) and the superior evaluation of the vascular wall itself without the use of intravascular contrast material. Involvement of the pericardium and (rarely) of the cardiac chambers can be diagnosed when the normally $2-3 \mathrm{~mm}$ wide curvilinear low signal intensity rim representing the pericardium is disrupted.

The advent of faster scanning techniques with spiral CT, electron beam CT (EBCT), and MDCT has considerably improved diagnostic accuracy of CT (fig. 1). The multiplanar imaging properties of MDCT and the excellent spatial resolution, in particuar, further increase the ability to assess invasion of mediastinal structures. EBCT is characterized by a very fast image acquisition of $200 \mathrm{~ms}$ but is limited by image noise. EBCT can freeze cardiac motion and should therefore be good at assessment of vascular involvement. Sensitivity and specificity values for EBCT have been reported to be $78 \%$ and $71 \%$, respectively, for the pulmonary artery, $70 \%$ and $71 \%$ for the carina, and $75 \%$ and $57 \%$ for the main bronchus [34]. Results should be superior with MDCT because of its better signal-to-noise ratio, especially if additional ECG gating is employed. However, performance was not yet quantified with this new technique.

Pleural involvement. Presence of a pleural effusion requires the exclusion of pleural involvement leading to stage IV and irresectability. An Italian group described a significantly higher signal enhancement in malignant pleural effusions in MRI after intravenous contrast application due to pathological diffusion processes [35].

The evaluation of pleural dissemination also profits from thin CT sections [32, 33]. Mori et al. [36] showed that the sensitivity could be increased from $50 \%$ (10/20 patients) to $90 \%$ with $2-\mathrm{mm}$ sections. Accuracy improved from $78 \%$ to $93 \%$. In 42 patients who underwent surgical resection, accuracy of thinsection CT was found to be $90 \%$ versus $83 \%$ with thick-section CT [36]. Overall sensitivity was 38\% and $75 \%$, respectively. Furthermore the sensitivity was 33\% and $100 \%$ at the interlobar fissure, $29 \%$ and $57 \%$ at the costal pleura, $25 \%$ and $25 \%$ at the mediastinal pleura and $20 \%$ and $60 \%$ at the diaphragm, respectively.

Transfissural tumour growth. Two studies evaluated the added value of multiplanar reconstructions based on thin sections (fig. 1). Spiral CT with thin sections was shown to be advantageous for evaluation of 
transfissural growth. STORTo et al. [37] demonstrated that the sensitivity of CT scanning for the presence of extension through the major fissure could be improved from $57 \%$ for thick $10-\mathrm{mm}$ sections to $87 \%$ with thin 2-mm sections. Additional multiplanar reformations (MPR) could further improve sensitivity to $100 \%$. For the minor fissure, 6 of 51 cases were inconclusive on axial section but only one remained so on MPRs.

UFFMANN et al. [38] could confirm these results when evaluating MDCT scanning for peripheral lung cancer. In $66 \%$ of cases, they found additional diagnostic information with $1.25 \mathrm{~mm}$ sections as compared to standard 5-mm sections. With MPR, evaluation was improved in an additional $38 \%$ of lesions. Segmental localization was altered in 5 of 24 patients when evaluation was based on thin axial sections, and it was further altered in an additional three patients when MPR was used. The assessment of pleural infiltration was altered in eight of 24 patients with thin sections and in an additional nine patients using MPR. They concluded that in patients with focal lung lesions the reconstruction of thin axial sections and MPR provides considerable additional diagnostic information and should become a diagnostic standard.

Chest wall involvement. Although chest wall and limited mediastinal invasion of lung cancer are no longer contraindications to surgical treatment, the mortality rate associated with en bloc resection of the tumour and the contiguous structures is still substantial. Therefore, information about chest wall and mediastinal invasion is still one of the most important factors in the clinical decision to perform surgery.

Both, CT and MRI have been used for the evaluation of tumour invasion of the chest wall. Gross extrapulmonary invasion does not pose problems, however, accurate distinction between tumour contiguity with adjacent extrapulmonary structures and actual invasion is often difficult on static images especially when no obvious soft tissue mass in the mediastinum or chest wall proves invasion. Contiguity of tumour with the parietal pleura was found not to be equivalent to definite invasion of the chest wall even when associated with a pleural thickening contiguous to the tumour.

Thin-section CT imaging (1-mm slices) was found to be superior to conventional CT techniques $(10-\mathrm{mm}$ slices) for the delineation of anatomical chest wall structures and thus for evaluating the presence of chest-wall involvement [39]. For the delineation of the extrapleural fat plane and the assessment of soft tissue structures a smoothing reconstruction algorithm was found to be superior to a high-resolution (HR) algorithm because of the increased noise level in HRCT sections.

Both, CT and MR have been used to apply dynamic imaging during different phases of respiration to assess the respiratory shift, that is defined as a change in the relative location between the peripheral lung tumour and the chest wall with deep inspiration and expiration. MRI uses coronal or sagittal T1-weighted breath-hold images that are obtained within $<30 \mathrm{~s}$ in inspiration and expiration. MDCT can be used to obtain 3D data sets in inspiration and expiration. In a similar fashion to MR, the evaluation then relies on coronal and sagittal images reconstructed from these data. Dynamic CT during a single expiratory manoeuvre over a time period of $10 \mathrm{~s}$ relies either on electron beam scanning $\left(200 \mathrm{~ms} \cdot\right.$ image $^{-1}$, one image every second [40]), or on continuous data acquisition using spiral CT scanners $\left(250-500 \mathrm{~ms} \cdot\right.$ image $\left.^{-1}\right)$. Such expiratory dynamic CT was found to provide accurate information in all 15 patients studied [40] but only proved to be helpful for tumours located in the middle and lower lobes.

It should also be noted that only absence of tumour invasion can be confidentially diagnosed; free movement of the tumour directly proves lack of attachment between the visceral and parietal pleura. Benign firm fibrous adhesion may cause false-positive results by simulating tumour infiltration. Such limitations hold true for CT and MRI as well as alternative approaches using ultrasound or pneumothorax CT. The latter is based on the idea that air in the pleural gap between the tumour and the chest wall proves the absence of tumour infiltration. However, this technique is invasive and may cause symptomatic pneumothorax (described in four of 43 patients) and therefore never found broad application [41].

3D images based on SSD were found to facilitate the differentiation between simple pleural tags (i.e. bands extending from the lesion to the visceral pleura) and pleural puckering (i.e. an indrawn locally thickened pleura) seen with visceral pleural invasion [42]. This study by KuriYAmA et al. [42] found that lesions classified as T1 on 2D axial CT images were correctly reclassified as T2 (visceral pleural invasion) or T3 (parietal pleural involvement) on 3D images in 10 of 42 patients. The sensitivity of pleural puckering was high, but specificity was only $76 \%$ because it was not only seen with tumour invasion but also with reactive fibrotic changes.

As a consequence, imaging is becoming an excellent tool for ruling out chest-wall invasion, but imaging remains bad at distinguishing patients in whom chestwall invasion is actually present from those with fibrous ashesions.

Superior sulcus tumours. Owing to its superior contrast resolution, MRI may demonstrate subtle chest-wall invasion and be superior to CT in this regard. The use of surface coils provides high-resolution images. Chestwall invasion is best depicted as a disruption of the extrapleural fat line (bright signal of fat in T1-weighted images). MRI was also thought to be more accurate than CT in depicting chest wall involvement from superior sulcus tumours with respect to involvement of the subclavian vessels, the brachial plexus anteriorly and the vertebra posteriorly. However, high quality spiral CT with thin sections, bolus injection of contrast agents and sagittal and coronal reformats provides the same information regarding the anatomical environment of the plexus, and MRI may be reserved for patients with extension into the neural foramina and the epidural space (fig. 2). 


\section{Nodal staging}

The evaluation of mediastinal lymph nodes is an important aspect of staging in patients with nonsmall cell lung cancer. The nodal status is an integral part of the TNM staging system and provides critical information for planning treatment and determining prognosis. The preoperative diagnosis of hilar lymph node metastases has become increasingly important in selecting patients for minimal resection.

CT serves not only as a basis for selecting those patients that have to undergo further invasive procedures (mediastinoscopy, mediastinotomy, thoracoscopy, transbronchial biopsy) for lymph node staging but also to select the appropriate procedure depending on the location of enlarged lymph nodes to be sampled. Despite the known inability of CT to detect microscopic metastases within normal sized lymph nodes, the exclusion of enlarged lymph nodes is sufficient to obviate further evaluation and have patients directly undergo thoracotomy. It is a common understanding, however, that enlarged lymph nodes seen in CT must be sampled with biopsy to avoid overstaging. Virtual bronchoscopy may be helpful to direct the most appropriate approach for transbronchial needle aspiration (see later).

Size criteria for mediastinal lymph node involvement. Traditionally imaging evaluation of mediastinal lymph node involvement has relied on anatomical features of the nodes, most notably nodal size. CT represents the imaging method of choice for determination of the tumour staging and it has been shown to be the best predictor of nodule volume. With regard to the determination of the nodal status, however, its limitation with relatively low sensitivity (41-67\%) and low specificity $(79-86 \%)$ are well documented based on the fact that enlarged lymph nodes may be hyperplastic rather than neoplastic and normal sized nodes may contain neoplastic cells [43].

Various approaches have been undertaken to refine the single morphological criterion of nodal size such as modulating the threshold size for various nodal locations within the mediastinum, or taking into account not only the smallest diameter as measured on the axial scan but also the shape of the lymph node (round versus oval).

Morphologic criteria for hilar lymph-node involvement. Shimoyama et al. [44] found an increased diagnostic accuracy to $88 \%$ using not only the size criterion ( $>10 \mathrm{~mm}$ in smallest diameter) but also morphological criteria for the assessment of hilar lymph-node involvement. Imaging was based on thin sections [44]. The authors found that in $95 \%$ of locations with normal lymph nodes there was a straight or concave pleural interface between hilar structures and lung parenchyma while in $95 \%$ of locations with malignant lymph nodes there was a convex interface. Application of this criterion lead to an improvement in sensitivity from $50 \%$ (for the size criterion) to $87 \%$ (for the morphological criterion). Specificity could be improved from $80 \%$ to $83 \%$.

Again the approach of considering size and shape of lymph nodes takes advantage of the availability of thin sections and high quality multiplanar images provided by MDCT (figs. 1 and 2). Unfortunately, no quantitative evaluation has been carried out so far. It is likely that refinement of morphological criteria will improve the diagnostic accuracy for evaluation of mediastinal lymph nodes. However, morphological criteria alone will most likely never be able to achieve the same diagnostic accuracy as imaging modalities that also take functional criteria into account, such as PET or MRI of the uptake of lymph node-specific contrast agents.

Magnetic resonance evaluation of lymph node involvement. MRI has no superiority over CT for the detection of mediastinal nodal involvement. MR used to be advocated for the evaluation of areas that would potentially benefit from multiplanar imaging capabilities such as the aortopulmonary window or the subcarinal region (figs. 1 and 4). This advantage, however, is completely ameliorated by the multiplanar imaging capabilities of thin-section spiral CT and MDCT that provide a far superior spatial resolution. Depending on scanner capabilities, the poorer spatial resolution of MR means that a group of distinct normal-sized lymph nodes may occasionally blur together and simulate the appearance of a single large nodal mass.

There was optimistic preliminary data suggesting that benign lymph nodes could be differentiated from malignant lymph nodes based on contrast enhancement characteristics. A significantly higher enhancement was found in malignant nodes after administration of gadolinium chelates (DOTA) as compared to little or no enhancement in benign nodes [45]. There was a significant overlap between malignant and inflammatory lymph nodes. However, these results could not be confirmed in larger patient series.

Lymph-node-specific magnetic resonance contrast agents. The introduction of new lymph node specific contrast agents is a promising development. Ultrasmall superparamagnetic iron oxide nanoparticles (USPIO) traverse the vascular endothelium and are phagocytosed by macrophages in normally functioning lymph nodes. This results in a uniform signal loss in T2 and T2* weighted images, which could be demonstrated in an animal model [46]. In patients with head and neck tumours, enlarged hyperplastic lymph nodes demonstrated a signal decrease similar to that encountered in normal sized lymph nodes. This allowed for differentiation of enlarged hyperplastic nodes from enlarged neoplastic lymph nodes [47]. Preliminary data in patients with bronchogenic carcinoma have shown a good sensitivity for the diagnosis of metastatic normal sized lymph nodes but unfortunately a relatively low specificity [48]. Currently, a multicentre trial is under way to determine efficacy, sensitivity and specificity for a larger number of patients.

False-positive results include benign reactive nodes with follicular hyperplasia. Most reactive nodes are filled with lymphocytes but contain few macrophages and thus exhibit only a low phagocytic activity and 
USPIO uptake. Localized nodal lipomatosis may account for a heterogeneous aspect of the node [49].

Positron emission tomography evaluation of lymph node involvement. PET relies on physiological rather than anatomical features for distinction between normal and neoplastic lymph nodes, and has the potential to demonstrate small neoplastic foci within normal sized nodes and to allow of enlarged hyperplastic from neoplastic nodes. However, PET may still be false negative in small malignant lymph nodes.

\section{Control after treatment/tumour recurrence}

CT remains the procedure of choice for routine follow-up and for patients with suspected tumour recurrence in the post-pneumonectomy patient. There was a considerable variation in the appearance of the post-pneumonectomy space ranging from complete obliteration in $27 \%$ of cases and a persisting fluid collection in the remaining $73 \%$. There was a superiority of MRI for the detection of soft tissue recurrence that showed a combination of mass effect and altered MR signal intensity. In fact any inhomogeneous signal in the pneumonectomy space should be suspicious for tumour recurrence [50].

Filling-in of previously patent ectatic bronchi occurred in 20 patients with proven recurrent disease. It was the first sign of recurrence in six patients. Eight patients with stable radiation-induced fibrosis and patent bronchi remained disease-free [51].

A problematic topic is the re-evaluation of the residual tumour and tumour activity after neoadjuvant chemotherapy to be followed by surgery. CT criteria tend to underestimate the therapeutic effect demonstrated by pathological examination. A $50 \%$ size reduction, a change in tumour morphology (round versus irregular), and the disappearance of contrast enhancement after application of intravenous contrast were suggested as criteria for complete response [52]. Similar criteria can also be employed for MRI. PET is an excellent tool to search for viable tumour tissue in patients in whom CT or MRI remain inconclusive.

\section{Role of virtual bronchoscopy and computed tomography bronchography}

Virtual bronchoscopy yields excellent displays of the tracheobronchial system (fig. 3). It has various advantages over fibreoptic bronchoscopy. It is much less invasive and is very well tolerated by the patients. It can display the bronchial system distal to a narrow stenosis. Inversion of the viewing direction of the virtual "bronchoscope" is possible and one is able to look towards the proximal portions of the tracheobronchial system [53]. Most important, however, is that all the additional information from the surrounding structures is available from the scans. Thus, there is information of the transmural extent of a tumour, the presence and location of lymph nodes and the location of suspicious areas within the lung parenchyma [52].
The main advantages of fibreoptic bronchoscopy are the options to perform biopsies of suspicious lesions and the information available from the colour and texture of the endobronchial surfaces. Virtual bronchoscopy with transparent walls or coloured extramural pathology may be used for guidelines or transbronchial biopsy [54].

In a clinical trial it was found that virtual bronchoscopy using volume rendering technique (VRT) improves the recognition of mild changes in airway calibre that are otherwise difficult to appreciate and the understanding of complex tracheobronchial anomalies [55].

\section{Summary}

Early detection of lung cancer may be improved by computer-aided detection schemes based on dualenergy imaging or temporal subtraction techniques. The most promising screening technique, however, involves low-dose CT. Differentiation between small $(<1 \mathrm{~cm})$ benign and malignant lesions can best be performed by evaluating tumour growth on follow-up scans using automated volumetric analysis. Lesions $<1 \mathrm{~cm}$ can be assessed by using tumour enhancement characteristics on CT or MRI, or by employing PET imaging.

T-staging of bronchogenic carcinoma still heavily depends on CT imaging. MDCT is the current technological standard, and can be expected to yield excellent results for assessment of transfissural tumour growth, pleural involvement, mediastinal, and chest wall invasion. MRI has advantages when MDCT is not available and may remain superior for the assessment of vascular invasion (fig. 4). Both techniques, however, are only able to positively exclude chest-wall invasion and to confirm it in extensive disease. The differentiation of adhesions from initial invasion remains a problem. PET scanning may provide superior results for detecting distant pleural spread.

$\mathrm{N}$-staging is notoriously insensitive when only a size criterion is used to differentiate benign from malignant nodules on CT or MRI. Additional morphological criteria have been shown to substantially improve the assessment of hilar lymph nodes and may further improve the evaluation of mediastinal nodes when MDCT is employed. PET, however, is at present the most promising imaging technique for mediastinal lymph node staging.

Virtual bronchoscopy can be used to direct biopsy of more peripheral pulmonary lesions or determine the optimum site for transbronchial biopsy. It allows for noninvasive assessment of anomalies of the central tracheobronchial system but cannot substitute for fibreoptic bronchoscopy in patients in which biopsy has to be performed or in which visual assessment of the mucosal surface is necessary.

\section{References}

1. Difacio $\mathrm{MC}$, MacMahon $\mathrm{H}, \mathrm{Xu} \mathrm{CW}$, et al. Digital chest radiography: effect of temporal subtraction 
images on detection accuracy. Radiology 1997; 202: 447-452.

2. Kido S, Ikezoe J, Naito H, et al. Clinical evaluation of pulmonary nodules with single exposure dual-energy subtraction chest radiography with an iterative noise reduction algorithm. Radiology 1995; 194: 407-412.

3. MacMahon H. Improvement in detection of pulmonary nodules: digital image processing and computeraided diagnosis. Radiographics 2000; 20: 1169-1177.

4. Prokop M. Multislice computed tomography of the lung parenchyma. In: Marincek J, Ros P, Reiser M, eds. Multislice CT - A Practical Guide. Proceedings of the 5th International Somatom CT Scientific User Conference. Springer, 2001; pp. 145-145.

5. Mastora I, Remy-Jardin M, Masson P, et al. Multidetector CT imaging of diffuse lung diseases: clinical impact of simultaneous reconstruction of contiguous thick and HRCT sections. Radiology 2000; 217(P): 384.

6. Vining DJ, Lin K, Choplin RH, Haponik E. Virtual bronchoscopy, relationships of virtual reality endobronchial simulations to actual bronchosopic findings. Chest 1996; 109: 549-553.

7. Hopper KD. CT Bronchoscopy. Semin Ultrasound CT MRI 1999; 20: 10-15.

8. Summers RM, Shaw DJ, Shelhamer JH. CT virtual bronchoscopy of simulated endobronchial lesions. Effect of scanning reconstruction and display settings and potential pitfalls. AJR 1998; 170: 947-950.

9. Schmidt M, Yang G, Keegan J, et al. Non-breath-hold lung magnetic resonance imaging with real time navigation. MAGMA 1997; 5: 123-128.

10. Lutterbey G, Gieseke J, Sommert T, et al. Ein neuer Ansatz in der Magnetresonanztomographie der Lunge mit einer ultrakurzen Turbo-spin-echo Sequenz (UTSE). RÖFO 1996; 164: 388-393.

11. Haddad JL, Rofsky NM, Ambrosino NM, Naidich DP, Weinreb JC. T2-weighted MR imaging of the chest. Comparison of ECG triggered conventional and turbo-spin-echo and non-triggered turbo spin-echo sequences. J Magn Reson Imaging 1995; 5: 325-329.

12. Bailes DR, Gilderale DJ, Bydder GM, Collins AG, Firmin DN. Respiratory ordered phase encoding (ROPE): a method for reducing respiratory motion artifacts in MR imaging. L Comput Assist Tomogr 1985; 9: 835-838.

13. Bergin C, Pauly J, Macovski A. Lung parenchyma: projection reconstruction MR imaging. Radiology 1991; 179: 777-781.

14. Bergin C, Glover G, Pauly J. Lung parenchyma: magnetic susceptibility in MR imaging. Radiology 1991; 180: 845-848.

15. Mayo J, MacKay A, Müller N. MR imaging of the lungs: value of short TE spin echo pulse sequences. AJR 1992; 159: 951-956.

16. Weissleder R, Elizondo G, Wittengerg J, Lee AS, Josephson L, Brady TJ. Ultrasmall superparamagnetic iron oxide: an intravenous contrast agent for assessing lymph nodes with imaging. Radiology 1990; 175: 494498.

17. Taupitz M, Barentsz JO, Tuerk I, et al. MR lymphography using USIOP: results of a clinical phase 3 trial in 31 patients with urological tumors. Radiology 1998; 209(P): 336.

18. Nishi J, Kadota M, Yamashita Y, et al. Detection of small lung nodules: the value of retrospective thin slice reconstruction and cine viewing with a multi-detector spiral system. Radiology 2000; 217(P): 384.

19. Diederich S, Lenzen H, Windmann R, et al. Low dose CT of pulmonary nodules: experimental and clinical studies. Radiology 1999; 213: 289-298.

20. Yankelevitz D, Reeves A, Kostis WJ, Zhao B, Henschke CJ. Small pulmonary nodules: volumetrically determined growth rates based on CT evaluation. Radiology 2000; 217: 251-256.

21. Henschke C, McCauley DI, Yankelevitz DF, et al. Early lung cancer project: overall design and findings from baseline screening. Lancet 1999; 354: 99-105.

22. Kaneko M, Eguchi K, Ohmatsu H, et al. Peripheral lung cancer: screening and detection with low dose spiral CT versus radiography. Radiology 1996; 201: 798-802.

23. Sone S, Takashima S, Li F, et al. Mass screening for lung cancer with mobile spiral computed tomography scanner. Lancet 1998; 351: 1242-1245.

24. Patz EF, Goodman PV, Bepler G. Screening for lung cancer. N Engl J Med 2000; 343: 1627-1633.

25. Kakinuma R, Ohmatsu H, Kaneko M, et al. Detection failures in spiral CT screening for lung cancer: analysis of CT findings. Radiology 1999; 212: 61-66.

26. Munden R, Pugatch R, Liptay M, Sugarbaker DJ, Le Lu. Small pulmonary lesions detected at CT: clinical importance. Radiology 1997; 202: 105-110.

27. Swensen SJ, Yamashita $\mathrm{K}$, McCollough $\mathrm{CH}$, et al. Lung nodules: dual-kilovolt peak analysis with CT multicenter study. Radiology 2000; 214: 81-85.

28. Swensen SJ, Viggiano RW, Midthun DE, et al. Lung nodule enhancement at CT: multicenter study. Radiology 2000; 214: 73-80.

29. Zhang M, Kono M. Solitary pulmonary nodules: evaluation of blood flow patterns with dynamic CT. Radiology 1997; 205: 471-478.

30. Hittmair K, Eckersberger F, Klepetko W, Helbich T, Herold C. Evaluation of solitary pulmonary nodules with dynamic contrast-enhanced MR imaging - a promising technique. Magn Reson Imag 1995; 13: 923-933.

31. Erasmus JJ, McAdams HP, Connolly JE. Solitary pulmonary nodules: evaluation of the indeterminate nodule. Radiographics 2000; 20: 59-66.

32. White PG, Adams H, Crane D, Butchart EG. Preoperative staging of carcinoma of the bronchus: can computed tomographic scanning reliably identify stage III tumors? Thorax 1994; 49: 951-957.

33. Quint LE, Francis IR, Wahl RL, Gross BH, Glazer GM. Preoperative staging of non-small cell carcinoma of the lung: imaging methods. AJR 1995; 164: 1349-1359.

34. Takahashi M, Shimoyama K, Murata K, et al. Hilar and mediastinal invasion of bronchogenic carcinoma: evaluation by thin-section electron beam computed tomography. J Thorac Imag 1997; 12: 195-199.

35. Padovani B, Mouroux J, Seksik L, et al. Chest wall invasion by bronchogenic carcinoma: evaluation with MR imaging. Radiology 1993; 187: 33-38.

36. Mori K, Hirose $\mathrm{T}$, Machida $\mathrm{S}$, et al. Spiral CT diagnosis of pleural dissemination in lung cancer: comparison of thick section and thin section spiral CT. J Thorac Imag 1998; 13: 211-218.

37. Storto M, Ciccotosto C, Guidotto A, Merlino B, Patea RL, Bonomo L. Neoplastic extension across pulmonary fissures: value of spiral CT and multiplanar reformations. J Thorac Imag 1998; 13: 204-210. 
38. Uffmann M, Prokop M, Schaefer-Prokop C, et al. Assessment of focal lung lesions with multislice CT: are thin sections and multiplanar reformats necessary? Eur Radiol 2001; 11: Suppl. B-0311.

39. Uhrmeister P, Allmann KH, Wertzel H, et al. Chest wall infiltration by lung cancer: value of thin sectional CT with different reconstruction algorithms. Europ Radiol 1999; 9: 1304-1309.

40. Murata K, Takahashi M, Mori M, et al. Chest wall and mediastinal invasion by lung cancer: evaluation with multisection expiratory dynamic CT. Radiology 1994; 191: 251-255.

41. Watanabe A, Shimokata K, Saka H, et al. Chest CT combined with artificial pneumothorax. Value in determining origin and extent of tumor. AJR 1991; 156: 707-710.

42. Kuriyama K, Tateishi R, Kumatani T, et al. Pleural invasion by peripheral bronchogenic carcinoma: assessment with three-dimensional spiral CT. Radiology 1994; 191: 365-369.

43. Gdeedo A, van Schil P, Corthouts B, van Mieghern F, van Meerbeeck J. Prospective evaluation of CT and mediastinoscopy in mediastinal lymph node staging. Eur Respir J 1997; 10: 1547.

44. Shimoyama K, Murata K, Takahashi M, Morita R. Pulmonary hilar lymph node metastases from lung cancer: evaluation based on morphology at thin section, incremental dynamic CT. Radiology 1997; 203: 187.

45. Laissy JP, Gay-Deapssier P, Soyer P, et al. Enlarged mediastinal lymph nodes in bronchogenic carcinoma: assessment with dynamic contrast enhanced MR imaging. Work in progress. Radiology 1994; 191: 263-267.

46. Vasallo P, Matei C, Heston WDW, et al. AMI-227 enhanced MR lymphography usefulness for differentiating reactive from tumor bearing lymph nodes. Radiology 1994; 193: 501-506.
47. Anzai Y, Blackwell KE, Hirschowitz SL, et al. Initial clinical experience with dextran-coated superparamagnetic iron oxide for detection of lymph node metastases in patients with head and neck cancer. Radiology 1994; 192: 709-715.

48. Bluemke DA, Borman TL, Wang K. MR lymph node contrast agent (Combidex): use in directing bronchoscopic fine needle aspiration of mediastinal lymph nodes. Radiology 1998; 209: 375.

49. Bellin MF, Beigelman C, Precetti-Morel S. Iron oxideenhanced MR lymphography: initial experience. Eur J Radiol 2000; 34: 257-264.

50. Heelan RT, Panicek DM, Burt ME, et al. Magnetic resonance imaging of the pneumonectomy chest. Normal and abnormal findings. J Thorac Imag 1976; 12: 200-208.

51. Libshitz HI, Sheppard DG. Filling in of radiation therapy-induced bronchiectatic change: a reliable sign of locally recurrent lung cancer. Radiology 1999; 210 : 25-27.

52. Seto M, Kuriyama K, Kasugai T, et al. Comparison of computed tomography and pathologic examination for evaluation of response of primary lung cancer to neoadjuvant therapy. $J$ Thorac Imag 1999; 14: 69-73.

53. Fleiter T, Merkle EM, Aschoff AJ, et al. Comparison of real time virtual and fiberoptic bronchoscopy in patients with bronchial carcinoma: opportunities and limitations. AJR 1997; 169: 1591-1595.

54. McAdams H, Goodman P, Kussin P, et al. Virtual bronchoscopy for directing transbronchial needle aspiration of hilar and mediastinal lymph nodes: a pilot study. AJR 1998; 170: 1361-1364.

55. Remy-Jardin M, Remy J, Artaud D, Fribourg M, Duhamel A. Volume rendering of the tracheobronchial tree: clinical evaluation of bronchographic images. Radiology 1998; 208: 761-770. 